\title{
Optimized Method to Generate Synthetically Challenging Macrocyclic Tetrapeptides That Do Not Have a Turn Inducer
}

\author{
Sanjeewa N. Senadheera ${ }^{1}$, and Jane V. Aldrich ${ }^{1,2}$ \\ ${ }^{I}$ Department of Medicinal Chemistry, The University of Kansas, Lawrence, KS, 66045, USA; \\ ${ }^{2}$ Department of Medicinal Chemistry, University of Florida, Gainesville, FL, 32610, USA
}

\section{Introduction}

Natural and synthetic cyclic peptides represent an important class of bioactive molecules that exhibit a broad range of biological activities [1] and are considered as promising lead compounds in the search for new drugs. In particular, macrocyclic tetrapeptides (MTPs) have potential for development into therapeutic agents because of their low molecular weight, small organic molecule-like properties, membrane permeability, selectivity, expected metabolic stability to proteases, and improved pharmacokinetic properties in vivo [1,2]. However, the small 12-membered ring size can make their synthesis difficult, often resulting in low yields of the desired MTP with resulting dimers and oliogomers as the major products [3-5]. Generally MTPs in which the amino acids are all the same chirality are more difficult to synthesize because the conformations of the linear precursor tetrapeptides limit the close approach of the $N$ - and $C$-termini needed for macrocyclization to occur [5]. Also to date, almost all reported synthesized MTPs contain at least one turn inducing residue such as Pro, an N-alkyl

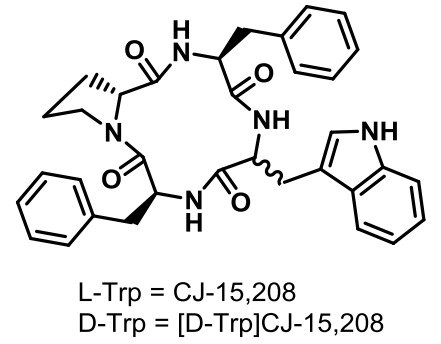

Fig. 1. Structure of CJ-15,208 and its D-Trp isomer [4]. amino acid, a D-amino acid or an unusual amino acid that favors macrocyclization $[1,6]$.

As part of our long standing interest in peptide ligands for kappa opioid receptors (KOR) we are synthesizing analogs of the natural product macrocyclic tetrapeptide CJ-15,208 (Figure 1) which exhibits modest affinity for KOR $[4,7]$. We use an improved synthetic protocol to prepare larger quantities of the macrocyclic tetrapeptide CJ-15,208, [D-Trp]CJ-15,208 and their analogs for detailed pharmacological evaluation in vivo [8-10]. To demonstrate the broad applicability of this methodology, here we describe the challenging syntheses of two all L-amino acid macrocyclic tetrapeptides (all L-AA MTPs, Figure 2) based on the CJ-15,208 scaffold that do not contain a turn inducer to promote macrocyclization.

\section{Results and Discussion}

The cyclic tetrapeptides were synthesized by a combination of solid phase synthesis of the linear peptide precursors on the 2-chlorotrityl chloride resin, followed by cyclization in solution [3,4,9]. The crucial cyclization step was optimized by modifying the reaction conditions reported previously (HATU, DIEA, DMF) [3,4].

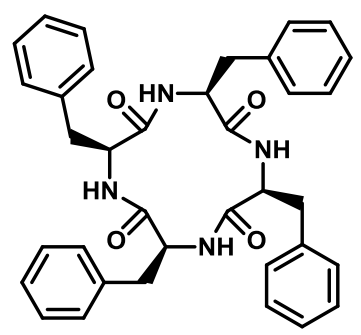

cyclo[Phe-Phe-Phe-Phe], 1

Fig. 2. Structure of an all L-AA MTP derived from CJ-15,208 that does not have a turn inducing residue. 
Table 1. Improvements in the isolated yield of all L-AA MTPs using the optimized protocol for synthesis and purification.

\begin{tabular}{cc}
\hline Peptide & $\begin{array}{c}\text { Yield of pure cyclic peptide using } \\
\text { the modified method }(\%)\end{array}$ \\
\hline cyclo[Phe-Phe-Phe-Phe], 1 & $15^{\mathrm{a}}$ \\
Analog of $\mathbf{1}$ & $40^{\mathrm{b}}$ \\
\hline
\end{tabular}

${ }^{a}$ Reported yield for MTP 1 was <1\% [11]; ${ }^{b}$ There is no previously reported synthesis for this all L-AA MTP.

In our optimized procedure we found that several experimental parameters have an influence on the yield in the crucial cyclization, namely the concentration and addition rate of the linear tetrapeptide to the reaction mixture, and the reaction temperature as well as reaction time. Depending on the particular tetrapeptide sequence these four experimental parameters for the cyclization step may have to be adjusted to generate the desired macrocyclic tetrapeptide in good yield. Accordingly, the addition rate of the linear peptide to the reaction and the concentration of the linear peptide were optimized to minimize dimer formation and to increase the yields. The temperature of the cyclization step was also slightly increased (from rt to $30-37^{\circ} \mathrm{C}$ after addition of the linear peptide). The crude MTPs were purified using normal phase column chromatography on silica gel. The syntheses of the all L-AA MTPs were successfully achieved in modest yields (Table 1). The all L-AA MTP cyclo[Phe-Phe-PhePhe], 1, was generated in significantly higher yield compared to the previously reported minimal yield of this cyclic peptide [11]. An analog of $\mathbf{1}$ which has not been previously prepared was obtained in substantially higher yield.

In conclusion, the use of these optimized conditions for the crucial cylization step permitted the synthesis of these all L-AA MTPs that do not contain a turn inducing residue. cyclo[Phe-Phe-PhePhe], 1, was obtained in substantially higher yield than the reported method (15\% vs. <1\% [11]), and an analog of 1 was also synthesized for the first time in good yield (40\%). This generally applicable optimized synthetic methodology could facilitate the development of a variety of synthetically challenging MTPs for potential therapeutic applications.

\section{Acknowledgments}

This research was supported by grant R01 DA018832 (National Institute on Drug Abuse).

\section{References}

1. White, C.J., Yudin, A.K. Nature 3, 509-524 (2011), http://dx.doi.org/10.1038/nchem.1062

2. Bockus, A.T., McEwen, C.M., Lokey, R.S. Curr. Top. Med. 13, 821-836 (2013), http://dx.doi.org/10.2174/1568026611313070005

3. Kulkarni, S.S., et al. Adv. Exp. Med. Bio. 611, 269-270 (2009), http://dx.doi.org/10.1007/978-0-387-73657$0 \_121$

4. Ross, N.C., et al. Tetrahedron Lett. 51, 5020-5023 (2010), http://dx.doi.org/10.1016/j.tetlet.2010.07.086

5. Haddadi, M.E., et al. J. Peptide Sci. 6, 560-570 (2000), http://dx.doi.org/10.1002/10991387(200011)6:11<560::AID-PSC275>3.0.CO;2-I

6. Fairweather, K.A., et al. Org. Lett. 12,3136-3139 (2010), http://dx.doi.org/10.1021/ol101018w

7. Ross, N.C., et al. Br. J. Pharmacol. 165, 1097-1108 (2012), http://dx.doi.org/10.1111/j.14765381.2011.01544.x

8. Aldrich, J.V., et al. J. Nat. Prod. 76, 433-438 (2013), http://dx.doi.org/10.1021/np300697k

9. Senadheera, S.N., et al. in Lebl, M. (Ed.) Peptides: Building Bridges (Proceedings of the $22^{\text {nd }}$ American Peptide Symposium), American Peptide Society, San Diego, 2011, pp. 346-347.

10. Aldrich, J.V., et al. Br. J. Pharmacol. 171, 3212-3222 (2014), http://dx.doi.org/10.1111/bph.12664

11. Cavelier-Frontin, F., et al. THEOCHEM 286, 125-130 (1993), http://dx.doi.org/10.1016/0166$\underline{1280(93) 87158-\mathrm{A}}$ 